\title{
Community-acquired lung respiratory infections in HIV-infected patients: microbial aetiology and outcome
}

\author{
Catia Cilloniz ${ }^{1,2}$, Antoni Torres ${ }^{1,2}$, Eva Polverino ${ }^{1,2}$, Albert Gabarrus ${ }^{1,2}$, \\ Rosanel Amaro ${ }^{1,2}$, Encarnacion Moreno ${ }^{1,2}$, Santiago Villegas ${ }^{3}$, Mar Ortega $^{4}$, \\ Josep Mensa ${ }^{4}$, Maria Angeles Marcos ${ }^{5}$, Asuncion Moreno ${ }^{4}$ and Jose M. Miro ${ }^{4}$
}

Affiliations: 'Dept of Pneumology, Institut del Tórax, Hospital Clinic, IDIBAPS, University of Barcelona, Barcelona, ${ }^{2}$ Centro de Investigación Biomédica En Red-Enfermedades Respiratorias (CIBERes), Barcelona, ${ }^{4}$ Infectious Disease Service, Hospital Clinic-IDIBAPS, University of Barcelona, Barcelona, and ${ }^{5}$ Dept of Microbiology, Barcelona Centre for International Health Research, Hospital Clinic, University of Barcelona, Barcelona, Spain. ${ }^{3}$ Departamento de Medicina Crítica y Cuidados Intensivos, Universidad CES, Medellin Colombia.

Correspondence: A. Torres, Dept of Pneumology, Hospital Clinic of Barcelona, Villarroel 170, Barcelona, Spain. E-mail: atorres@clinic.ub.es

ABSTRACT We describe the aetiology of community-acquired pneumonia (CAP) in HIV-infected patients, risk factors for bacterial or Pneumocystis jirovecii CAP and prognostic factors of 30-day mortality.

This was a prospective observational study of 331 consecutive adult CAP cases in HIV-infected patients (January 2007 to July 2012).

$128(39 \%)$ patients had $\mathrm{CD}^{+}$cell counts $<200$ per $\mathrm{mm}^{3}$ and $99(43 \%)$ ha HIV RNA levels $<200$ copies per $\mathrm{mL}$ on antiretroviral therapy. Streptococcus pneumoniae was the most frequent microorganism in the group with $\mathrm{CD}^{+}$cell counts $\geqslant 200$ per $\mathrm{mm}^{3} ;$ P. jirovecii was the most frequent microorganism in the group with $\mathrm{CD}^{+}$cell counts $<200$ per $\mathrm{mm}^{3}$ and in patients with HIV RNA $\geqslant 200$ copies per $\mathrm{mL}$. Predictors of bacterial CAP were: time with symptoms $\leqslant 5$ days (OR 2.6, 95\% CI 1.5-4.4), C-reactive protein level $\geqslant 22 \mathrm{mg} \cdot \mathrm{dL}^{-1}$ (OR 4.3, 95\% CI 2.3-8.2) and hepatitis C virus co-infection (OR 2.3, 95\% CI 1.4-3.9). White blood cell count $\leqslant 4 \times 10^{12}$ per L (OR 3.7, 95\% CI 1.2-11.5), lactate dehydrogenase (LDH) level $\geqslant 598 \mathrm{U} \cdot \mathrm{L}^{-1}$ (OR 12.9, 95\% CI 4.2-39.7) and multilobar infiltration (OR 5.8, 95\% CI 1.9-19.5) were predictors of P. jirovecii. Overall 30-day mortality was 7\%. Appropriate antibiotic treatment (OR 0.1, 95\% CI $0.03-0.4), \mathrm{LDH} \geqslant 598 \mathrm{U} \cdot \mathrm{L}^{-1}$ (OR 6.2, 95\% CI 1.8-21.8) and mechanical ventilation (OR 22.0, 95\% CI 6.2-78.6) were the variables independently associated with 30 -day mortality.

The described predictors may help clinicians to distinguish between bacterial and P. jirovecii pneumonia in patients with suspected or confirmed HIV infection.

@ERSpublications

Clinical risk factors in HIV patients to distinguish between bacterial and Pneumocystis jirovecii pneumonia http://ow.ly/sV2hf

This article has supplementary material available from www.erj.ersjournals.com

Received: Sept 052013 | Accepted after revision: Jan 022014 | First published online: Feb 132014

Support statement: This work was supported by Ciber de Enfermedades Respiratorias (grant CB06/06/0028), 2009 Support to Research Groups of Catalonia 911 and IDIBAPS.

Conflict of interest: None declared.

Copyright @ERS 2014 


\section{Introduction}

Community-acquired pneumonia (CAP) is a frequent respiratory complication in HIV patients even in the highly active antiretroviral therapy (HAART) era [1-3]. Patients infected with HIV are 25 times more likely to develop pneumonia. The depletion of $\mathrm{CD}^{+}$lymphocytes and high levels of HIV RNA in HIV-infected persons occur in parallel to the risk of developing pulmonary infections [4]. It is well known that CD4 ${ }^{+}$ levels $<200$ cells per $\mathrm{mm}^{3}$ are a risk factor for Pneumocystis jirovecii pneumonia (PCP) [5-7]. However, there is no information regarding HIV RNA levels. A frequent clinical problem in HIV-infected patients is the clinical difficulty in distinguishing between bacterial CAP and PCP. In the most severe cases, clinicians combine antibacterial therapy and P.jirovecii coverage until microbiological results are available. The situation is even more difficult when a bronchoalveolar lavage cannot be performed due to respiratory failure. In patients with undiagnosed HIV, P. jirovecii may be ignored and, consequently, these patients may not receive adequate empirical therapy. Accordingly, clinical criteria to help clinicians in choosing antimicrobial therapy are needed.

The aims of this study were to describe the clinical and microbial characteristics of consecutive HIV patients with CAP, to describe the associated risk factors for bacterial pneumonia and PCP, and to assess 30-day mortality prognostic factors.

\section{Methods \\ Study design and patients}

This prospective observational study was carried out in the Hospital Clinic, Barcelona, Spain. All consecutive cases of adult patients with HIV infection and CAP between January 2007 and July 2012 were included. CAP was defined as the presence of a new infiltrate on chest radiography together with clinical signs/symptoms suggestive of lower respiratory tract infection. Mycobacterial and fungal infections (other than P.jirovecii) were also recorded but not included in the analysis.

\section{Data collection}

A series of clinical and biological data were collected on admission and during patients' hospitalization (online supplementary material). HIV tests were performed in all suspected cases (patients with HIV risk factors, or with presumed or confirmed PCP). Secondary PCP prophylaxis was implemented after cure of the current episode of PCP. All surviving patients were visited or contacted by telephone within 30 days of discharge. This study was approved by the Ethics Committees, Hospital Clinic (number 2009/5451). Patient identification remained anonymous and the need for informed consent was waived due to the observational nature of the study.

\section{Definition}

The categorical $\mathrm{CD}^{+}$cell count was expressed using the cut-offs $<200$ and $\geqslant 200$ cells per $\mathrm{mm}^{3}$. The categorical plasma HIV RNA load was expressed using the cut-offs: $<200$ and $\geqslant 200$ copies per mL. All patients with undetectable HIV RNA $(<200$ copies per $\mathrm{mL}$ ) were on effective HAART. "Late presenters" were considered those patients presenting pneumonia with a CD $4^{+}$count $<350$ cells per $\mathrm{mm}^{3}$ at the time of HIV diagnosis [8].

Appropriateness of empirical antibiotic treatment in all patients was defined according to Infectious Disease Society of America/American Thoracic Society guidelines [9].

\section{Microbiological evaluation}

Microbiological investigation was performed on sputum, urine, two blood samples and nasopharyngeal swabs (online supplementary material). Pleural puncture, tracheobronchial aspirates and bronchoalveolar lavage fluid, when available, were collected for Gram, Ziehl-Nielsen, May-Grünwald-Giemsa and Gomori methenamine silver stains, and for cultures of bacterial, fungal and mycobacterial pathogens.

Sputum and blood samples were obtained for bacterial culture before start of antibiotic therapy in the emergency department. Nasopharyngeal swabs for respiratory virus detection and urine samples for Streptococcus pneumoniae and Legionella pneumophila antigen detection were obtained within $24 \mathrm{~h}$ of hospital admission. Blood samples for serology of atypical pathogens and respiratory virus were performed at admission and 4-6 weeks thereafter (online supplementary material).

For the diagnosis of $P$. jirovecii infection we used Gomori methenamine silver stain in respiratory samples. Viral load was determined by Versant HIV-1 RNA 1.0 kPCR (Siemens Diagnostics, Munich, Germany) (lower limit of quantification: 37 copies per mL; upper limit of quantification: 11000000 copies per mL) (lower limit of quantification in the period 2007-2009: $<50$ copies per mL; 2010-2012: $<37$ copies per mL). 


\section{Statistical analysis}

Data are presented as $\mathrm{n}(\%)$ for categorical variables and median (interquartile range (IQR)) for continuous variables with non-normal distribution or mean $\pm \mathrm{SD}$ for those with normal distribution. Categorical variables were compared using the Chi-squared test or Fisher's exact test. Univariate and multivariate logistic regression analyses were performed to identify variables predictive of 30-day mortality (dependent variable). Variables that showed a significant result in the univariate analysis $(p<0.1)$ were included in the multivariate logistic regression backward stepwise model. Also, univariate and two multivariate logistic regression models were performed to predict bacterial CAP and PCP, respectively. Highly correlated variables were excluded from multivariate analyses. The Hosmer-Lemeshow goodness-of-fit test was performed to assess the overall fit of the model [10]. The level of significance was set at $\mathrm{p}=0.05$ (two-tailed). All analyses were performed using SPSS Statistics 18.0 (IBM, Armonk, NY, USA).

\section{Results}

\section{General patient characteristics}

During the study period (January 2007 to July 2012), a total of 1985 patients with CAP were admitted to our hospital; 1654 were not HIV infected and were excluded. Our study cohort consisted of 331 (17\%) HIVinfected patients hospitalised with a diagnosis of CAP; all these patients were infected by HIV-1. During the same study period, 30 cases of pulmonary tuberculosis and seven fungal respiratory infections (four histoplasmoses, two cryptococcoses and one aspergillosis) were recorded as community-acquired infections in HIV-infected patients but they were not included in our analysis. The main clinical characteristics of the study population are summarised in table 1 .

There were $243(73 \%)$ males with a mean \pm SD age of $42.1 \pm 9.5$ years. A total of $278(84 \%)$ patients admitted were Spanish and $53(16 \%)$ were from other countries. $320(97 \%)$ patients were hospitalised (63 (19\%) in intensive care unit (ICU)) and eight (2\%) were treated as outpatients. 59 (18\%) patients had received previous antibiotic treatment and $134(41 \%)$ patients received cotrimoxazole as PCP prophylaxis.

HIV infection had been diagnosed prior to hospital admission in $274(83 \%)$ patients and 170 (51\%) were on HAART. 57 (17\%) patients were diagnosed with HIV infection during the pneumonia episode (within the first 5 days after admission) and were predominantly late presenters $(n=46,81 \%)$ and more likely to suffer a $P$. jirovecii infection in comparison with those diagnosed of HIV before pneumonia (35\% versus $8 \%$ ) (table 2).

The majority of pulmonary infiltrates $(n=225,68 \%)$ were lobar alveolar opacities, followed by interstitial $(\mathrm{n}=94,28 \%)$ and mixed alveolar-interstitial $(\mathrm{n}=12,4 \%)$ patterns. Most pneumonia episodes were confined to one lobe $(n=198,60 \%)$ and the lower lobes were predominantly affected. Pleural effusion was present in 35 $(11 \%)$ cases and empyema in eight $(2 \%)$ cases, while cavitations were described in six (2\%) cases.

$140(42 \%)$ patients were co-infected with hepatitis C virus (HCV) and 31 (9\%) with hepatitis B virus.

The mean $\pm \mathrm{SD} \mathrm{CD} 4^{+}$cell count was $281.3 \pm 248.3$ per $\mathrm{mm}^{3}$ (median 240 cells per $\mathrm{mm}^{3}$, IQR $69-400$ cells per $\left.\mathrm{mm}^{3}\right) .128(39 \%)$ patients had $\mathrm{CD}^{+}$cell counts $<200$ per $\mathrm{mm}^{3}$. The mean HIV RNA level was $236108 \pm 905528$ copies per mL (median 10300 copies per mL, IQR 61-161000 copies per mL). 99 (30\%) patients had HIV RNA levels $<200$ copies per $\mathrm{mL}$.

An aetiological diagnosis was achieved in 227 (69\%) cases. Monomicrobial infection was detected in 189 $(83 \%)$ cases and polymicrobial in $38(17 \%)$ cases. The most frequently identified pathogens were S. pneumoniae ( $\mathrm{n}=100,30 \%$; more frequent among patients diagnosed with HIV prior to pneumonia), $P$. jirovecii ( $\mathrm{n}=42,13 \%$; more frequent among patients diagnosed with HIV during hospitalisation), mixed aetiology $(n=38,11 \%)$, respiratory viruses $(18,5 \%)$, Haemophilus influenzae $(n=7,2 \%)$ and Staphylococcus aureus ( $\mathrm{n}=6,2 \%)$ (fig. 1 and table 2$)$.

Several differences in radiological features between pneumonias with different aetiological microorganism were observed (online supplementary table 3). P. jirovecii and respiratory viruses were the most frequently identified pathogens in cases of the interstitial pattern $(\mathrm{p}<0.001$ each) and S. pneumoniae in the alveolar pattern $(\mathrm{p}<0.001)$. Multilobar involvement was more often associated with S. pneumoniae $(\mathrm{p}=0.025)$, $P$. jirovecci $(\mathrm{p}<0.001)$ and $S$. aureus cases $(\mathrm{p}=0.030)$. Pleural effusion was more frequent in pneumococcal pneumonia $(37 \%)$ than for other aetiologies, although this difference was not significant. S. aureus was detected in $50 \%$ of all cases of pulmonary cavitations.

Only five (5\%) out of 100 cases of pneumococcal pneumonia had received the pneumococcal vaccine in the previous five years.

Among the 42 patients with PCP diagnosis, 40 were started on HAART (95\%). Six patients died and two of them did not receive HAART therapy. The median (IQR) time from admission to HAART therapy among 
TABLE 1 Baseline characteristics of the whole population and of subgroups according to the time of HIV diagnosis

\begin{tabular}{|c|c|c|c|c|}
\hline Variables & Total population & $\begin{array}{l}\text { HIV diagnosis prior to } \\
\text { hospitalisation }\end{array}$ & $\begin{array}{l}\text { HIV diagnosis during } \\
\text { hospitalisation }\end{array}$ & p-value ${ }^{\#}$ \\
\hline \multicolumn{4}{|l|}{ Demographics } & \\
\hline Age years mean $\pm S D$ & $42.1 \pm 9.5$ & $42.7 \pm 9.1$ & $39.4 \pm 10.7$ & 0.017 \\
\hline Males & $243(73.4)$ & $193(70.4)$ & $50(87.7)$ & 0.007 \\
\hline Current smoking & 220 (66.9) & $192(70.6)$ & $28(49.1)$ & 0.002 \\
\hline Influenza vaccine & $20(7.2)$ & $19(8.4)$ & $1(2.0)$ & 0.11 \\
\hline Pneumococcal vaccine & $17(6.2)$ & $17(7.6)$ & $0(0)$ & 0.042 \\
\hline Time with symptoms days median (IQR) & $5.0(3.0-7.0)$ & $5.0(3.0-7.0)$ & $7.0(5.0-14.0)$ & 0.001 \\
\hline HAART & $170(51.4)$ & $165(60.2)$ & $5(8.8)$ & $<0.001$ \\
\hline Cotrimoxazole prophylaxis & $134(40.5)$ & $130(47.4)$ & $4(7.0)$ & $<0.001$ \\
\hline Co-infection with HCV & $140(42.3)$ & $130(47.4)$ & 10 (17.5) & $<0.001$ \\
\hline Chronic cardiovascular disease & $9(2.7)$ & $7(2.6)$ & $2(3.5)$ & 0.69 \\
\hline Diabetes mellitus & $14(4.3)$ & $13(4.8)$ & $1(1.8)$ & 0.30 \\
\hline Neurological disease & $89(27.1)$ & $82(30.1)$ & 7 (12.3) & 0.006 \\
\hline Chronic renal disease & $16(4.8)$ & $15(5.5)$ & $1(1.8)$ & 0.23 \\
\hline Chronic liver disease & $108(32.6)$ & $100(36.5)$ & $8(14.0)$ & 0.001 \\
\hline $\mathrm{CD4}^{+}$cells per $\mathrm{mm}^{3}$ & & & & $<0.001$ \\
\hline$<200$ & $128(38.7)$ & $86(31.4)$ & $42(73.7)$ & \\
\hline$\geqslant 200$ & 203 (61.3) & $188(68.6)$ & 15 (26.3) & \\
\hline Plasma HIV RNA copies per mL & & & & $<0.001$ \\
\hline$<200$ & 99 (29.9) & $96(35.0)$ & $3(5.3)$ & \\
\hline$\geqslant 200$ & $232(70.1)$ & $178(65.0)$ & $54(94.7)$ & \\
\hline
\end{tabular}

Data are presented as $\mathrm{n}(\%)$, unless otherwise stated. Percentages calculated from nonmissing data. IQR: interquartile range; HAART: highly active retroviral therapy; HCV: hepatitis C virus; HBV: hepatitis B virus. ${ }^{\#}$ : for comparisons between HIV diagnosis prior to and during hospitalisation; ": persons presenting with pneumonia with a $\mathrm{CD}^{+}$count $<350$ cells per $\mathrm{mm}^{3}$. Bold indicates statistical significance.

TABLE 2 Microbial aetiology in the whole HIV population ( $n=331$ ) and subgroups according to the time of HIV diagnosis

\begin{tabular}{|c|c|c|c|c|}
\hline Microorganisms & Total population & $\begin{array}{l}\text { HIV diagnosis prior to } \\
\text { hospitalisation }\end{array}$ & $\begin{array}{l}\text { HIV diagnosis during } \\
\text { hospitalisation }\end{array}$ & p-value \\
\hline Subjects $n$ & 331 & 274 & 57 & \\
\hline Streptococcus pneumoniae & $100(30.2)$ & 90 (32.8) & 10 (17.5) & 0.022 \\
\hline Pneumocystis jirovecii & $42(12.7)$ & $22(8.0)$ & $20(35.1)$ & $<0.001$ \\
\hline Mixed aetiology $\pi$ & 38 (11.5) & $28(10.2)$ & $10(17.5)$ & 0.11 \\
\hline Staphylococcus aureus & $6(1.8)$ & $6(2.2)$ & $0(0)$ & 0.59 \\
\hline Pseudomonas aeruginosa & $4(1.2)$ & $4(1.5)$ & $0(0)$ & $>0.99$ \\
\hline Legionella pneumophila & $3(0.9)$ & $3(1.1)$ & $0(0)$ & $>0.99$ \\
\hline Escherichia coli & $1(0.3)$ & $1(0.4)$ & $0(0)$ & $>0.99$ \\
\hline Klebsiella pneumoniae & $1(0.3)$ & $1(0.4)$ & $0(0)$ & $>0.99$ \\
\hline Mycoplasma pneumoniae & $1(0.3)$ & $0(0)$ & $1(1.8)$ & 0.17 \\
\hline
\end{tabular}




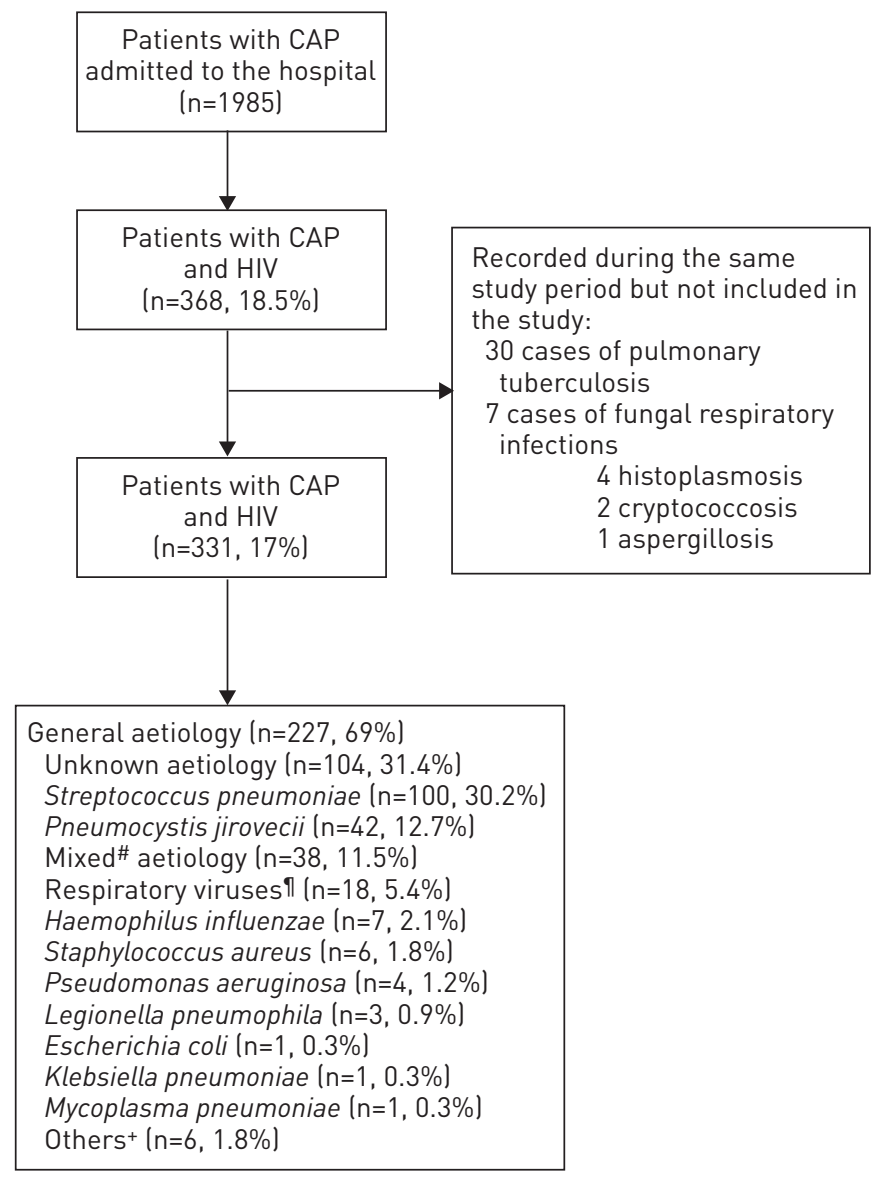

FIGURE 1 Flow chart of general aetiology. CAP: community-acquired pneumonia. \#: Streptococcus pneumoniae plus influenza virus A, Haemophilus influenzae plus influenza virus A, S. pneumoniae plus adenovirus, S. pneumoniae plus rhinovirus, Legionella pneumophila plus rhinovirus or S. pneumoniae plus Pseudomonas aeruginosa; ${ }^{\uparrow}$ : influenza virus A or B, rhinovirus, adenovirus, respiratory syncytial virus, or parainfluenza virus 2 or 3 ; $^{+}$: Enterococcus faecalis, Streptococcus sanguis, Streptococcus constellatus, Fusobacterium or Streptococcus pyogenes.

survivors and nonsurvivors patients was $15.5(10-22.5)$ and $15.5(9.5-16)$ days, respectively $(\mathrm{p}=0.41)$. In the second part of the study, HAART therapy was started earlier (14 (8-21) days in 2010-2012 versus 16 (14-32) days in 2007-2009, $\mathrm{p}=0.60$ ).

The median (IQR) length of hospital stay was 7.0 (4.0-13.0) days (table 3). The majority of patients (74\%) were classified as low risk (Pneumonia Severity Index class I-III). The most frequent complications were bacteraemia $(n=50,15 \%)$ and pleural effusion $(n=35,11 \%) .63(19 \%)$ patients were admitted to the ICU and $49(15 \%)$ of these required mechanical ventilation. The overall 30 -day mortality was $7 \%$ and was, globally, slightly higher for infections caused by H. influenzae (one (14\%) out of seven isolates) and P. jirovecii (six (14\%) out of 42), while it was only $4 \%$ (four out of 100 ) for pneumococcal infection, $8 \%$ (three out of 38) for mixed aetiology, 11\% (two out of 18) for respiratory viruses and one fatality case was described for Escherichia coli (one isolate).

By comparing patients with PCP with those with bacterial infection, we observed that they were less likely to be current smokers and on chronic treatment with HAART therapy or cotrimoxazole prophylaxis, and to show lower levels of C-reactive protein or leukocyte counts, but described more days of pneumonia-like symptoms prior to hospitalisation, more frequent previous antibiotic therapy, multilobar infiltration on radiography, hypoxaemia, higher lactate dehydrogenase (LDH) levels and worse outcomes (table 4).

\section{Predictors of bacterial CAP}

Using receiver operating characteristic (ROC) analysis, the optimal cut-point for C-reactive protein level was $22 \mathrm{mg} \cdot \mathrm{dL}^{-1}$, with $35 \%$ sensitivity, $87 \%$ specificity, $71 \%$ predictive positive value, $60 \%$ predictive negative value, positive likelihood ratio 2.75 and negative likelihood ratio 0.75 , with area under the curve (AUC) 0.65 (95\% CI $0.58-0.71)$, and for $\mathrm{LDH}$ was $598 \mathrm{U} \cdot \mathrm{L}^{-1}$, with $80 \%$ sensitivity, $37 \%$ specificity, $54 \%$ 
TABLE 3 Clinical presentation and outcomes

\begin{tabular}{|c|c|c|c|c|}
\hline Variables & Total population & $\begin{array}{l}\text { HIV diagnosis prior to } \\
\text { hospitalisation }\end{array}$ & $\begin{array}{l}\text { HIV diagnosis during } \\
\text { hospitalisation }\end{array}$ & p-value ${ }^{\#}$ \\
\hline Creatinine $\mathrm{mg} \cdot \mathrm{dL}^{-1}$ & $0.9(0.8-1.2)$ & $0.9(0.8-1.2)$ & $1.0(0.8-1.4)$ & 0.18 \\
\hline$\geqslant 1.5$ & $54(16.4)$ & $41(15.0)$ & $13(23.2)$ & 0.13 \\
\hline C-reactive protein $\mathrm{mg} \cdot \mathrm{dL}^{-1}$ & $11.3(5.0-21.0)$ & $12.1(5.1-21.6)$ & $9.0(4.0-19.0)$ & 0.32 \\
\hline WBCs $\times 10^{9}$ per $L$ & $8255(5150-11850)$ & 8850 (5400-11995) & 7000 (4650-10 755) & 0.065 \\
\hline$>4000$ & $277(84.5)$ & $232(85.3)$ & $45(80.4)$ & 0.35 \\
\hline Platelets $\times 10^{9}$ per $\mathrm{L}$ & $207.0(136.0-300.0)$ & 203.5 (135.5-289.5) & $245.0(151.0-339.0)$ & 0.52 \\
\hline$\geqslant 400$ & $16(7.5)$ & $15(8.5)$ & $1(2.7)$ & 0.22 \\
\hline $\mathrm{LDH} U \cdot \mathrm{L}^{-1}$ & $400.0(337.0-657.0)$ & $347.5(327.0-545.0)$ & $670.0(409.0-935.0)$ & $<0.001$ \\
\hline$\geqslant 598$ & $95(28.7)$ & $61(22.3)$ & $34(59.6)$ & $<0.001$ \\
\hline Bacteraemia & $50(15.2)$ & $41(15.0)$ & $9(15.8)$ & 0.88 \\
\hline Pleural effusion & $35(10.6)$ & $31(11.3)$ & $4(7.0)$ & 0.34 \\
\hline ICU admission & $63(19.0)$ & $46(16.8)$ & $17(29.8)$ & 0.023 \\
\hline Mechanical ventilation & $49(15.4)$ & $43(16.3)$ & $6(10.9)$ & 0.31 \\
\hline Septic shock & $22(6.7)$ & $19(7)$ & $3(5.4)$ & 0.65 \\
\hline Length of hospital stay days & $7.0(4.0-13.0)$ & $7.0(4.0-13.0)$ & $10.0(5.0-17.0)$ & 0.045 \\
\hline 30-day mortality & $22(6.6)$ & $19(6.9)$ & $3(5.3)$ & 0.65 \\
\hline
\end{tabular}

predictive positive value, $66 \%$ predictive negative value, positive likelihood ratio 1.27 and negative likelihood ratio 0.54, with AUC 0.56 (95\% CI 0.50-0.63) (online supplementary fig. 1).

Several variables were significantly associated with bacterial CAP at the univariate logistic regression analysis (table 5). Among these variables, $\leqslant 5$ days of symptoms (OR 2.6, 95\% CI 1.5-4.4), cotrimoxazole prophylaxis (OR 2.0, 95\% CI 1.2-3.4), C-reactive protein level $\geqslant 22 \mathrm{mg} \cdot \mathrm{dL}^{-1}$ (OR 4.3, 95\% CI 2.3-8.2) and HCV co-infection (OR 2.3, 95\% CI 1.4-3.9) were identified as risk factors for bacterial CAP in the multivariate analysis. The goodness-of-fit of the model tested using the Hosmer-Lemeshow test revealed adequate model fit $(\mathrm{p}=0.89)$.

\section{Predictors of PCP}

There was no association between HAART therapy and CAP aetiology, excepting those patients without HAART therapy that presented higher percentages of PCP $(20 \%$ versus $5 \%, \mathrm{p}<0.001)$. Only five $(12 \%)$ patients with PCP received cotrimoxazole therapy. This is because, in most cases, the diagnosis of HIV infection was done at the time of hospital admission or because HIV-infected patients had poor adherence to PCP prophylaxis.

Using ROC analysis, the optimal cut-point for C-reactive protein level was $12 \mathrm{mg} \cdot \mathrm{dL}^{-1}$, with $74 \%$ sensitivity, $53 \%$ specificity, $19 \%$ predictive positive value, $93 \%$ predictive negative value, positive likelihood ratio 1.57 and negative likelihood ratio 0.49, with AUC 0.65 (95\% CI $0.57-0.73$ ), and for LDH was $598 \mathrm{U} \cdot \mathrm{L}^{-1}$, with $86 \%$ sensitivity, $80 \%$ specificity, $38 \%$ predictive positive value, $97 \%$ predictive negative value, positive likelihood ratio 4.20 and negative likelihood ratio 0.18, with AUC 0.84 (95\% CI 0.77-0.91) (online supplementary fig. 1).

Several variables were significantly associated with PCP in the univariate and multivariate logistic regression analyses (table 6). Among these variables, we found the following independent predictors for PCP: female sex (OR 0.2, 95\% CI 0.1-0.9), current smoking (OR 0.4, 95\% CI 0.1-0.9), cotrimoxazole prophylaxis (OR $0.1,95 \%$ CI $0.04-0.5$ ), white blood cell (WBC) count $\leqslant 4 \times 10^{12}$ per L (OR 3.7, 95\% CI 1.2-11.5), LDH 
TABLE 4 Characteristics of pneumonia cases stratified by microbial aetiology

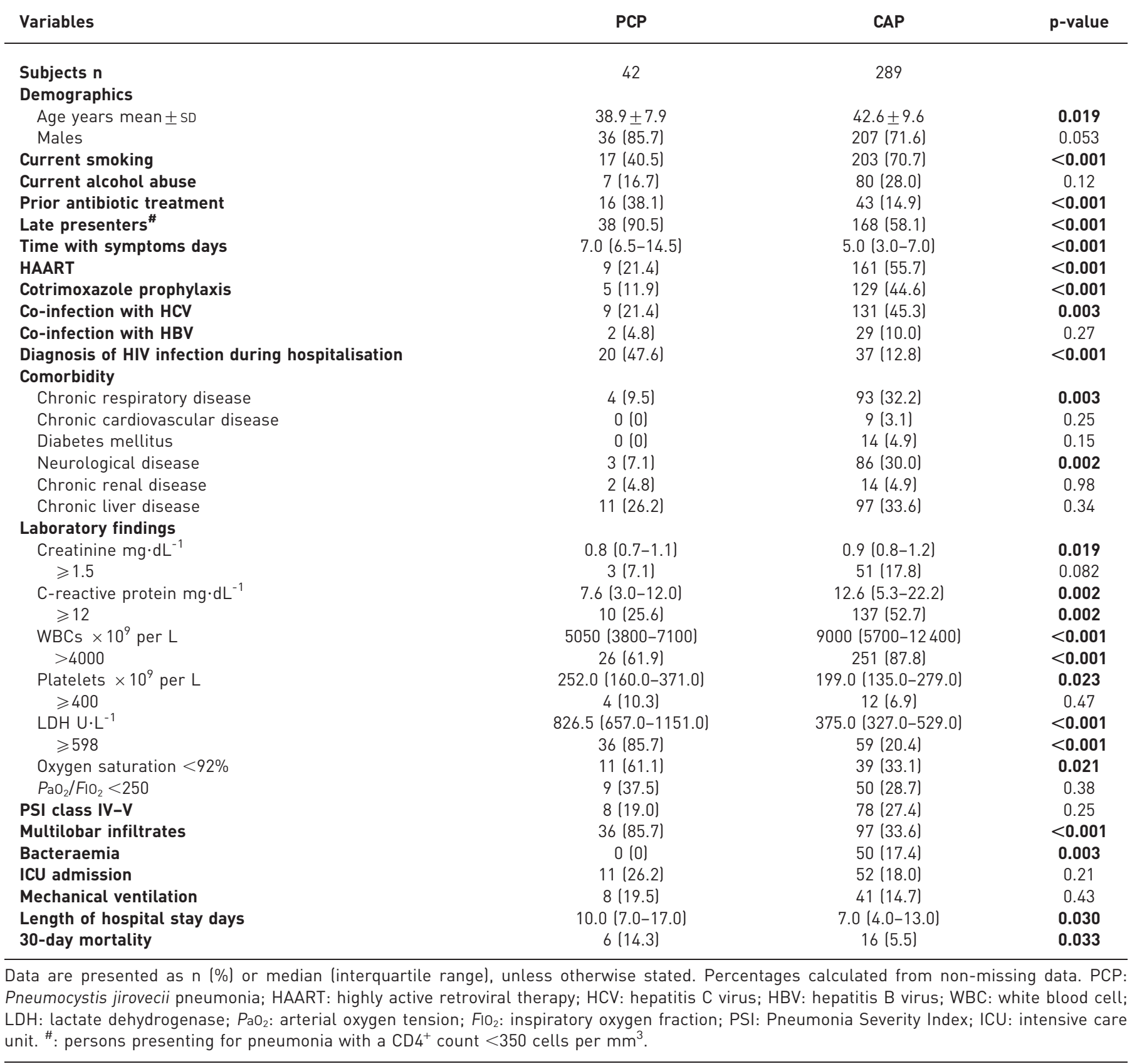

$\geqslant 598 \mathrm{U} \cdot \mathrm{L}^{-1}$ (OR 12.9, 95\% CI 4.2-39.7) and multilobar infiltration (OR 5.8, 95\% CI 1.9-19.5). The model was well calibrated with a p-value in the Hosmer-Lemeshow test 0.21 .

The combination of both multilobar involvement and $\mathrm{LDH} \geqslant 598 \mathrm{U} \cdot \mathrm{L}^{-1}$ for the prediction of PCP had a $76 \%$ sensitivity, $89 \%$ specificity, $51 \%$ predictive positive value, $96 \%$ predictive negative value, positive likelihood ratio 7.10 and negative likelihood ratio 0.27 .

Mortality and predictors of 30-day mortality

The overall 30 -day mortality rate was $7 \%(n=22)$. PCP was the most frequent isolate in nonsurvivors and differed from survivors $(12 \%$ versus $27 \%, \mathrm{p}=0.033$ ); the rest of the microorganisms did not differ between survivors and nonsurvivors. 
TABLE 5 Significant univariate and multivariate logistic regression analyses of predictors for bacterial community-acquired pneumonia in the HIV population

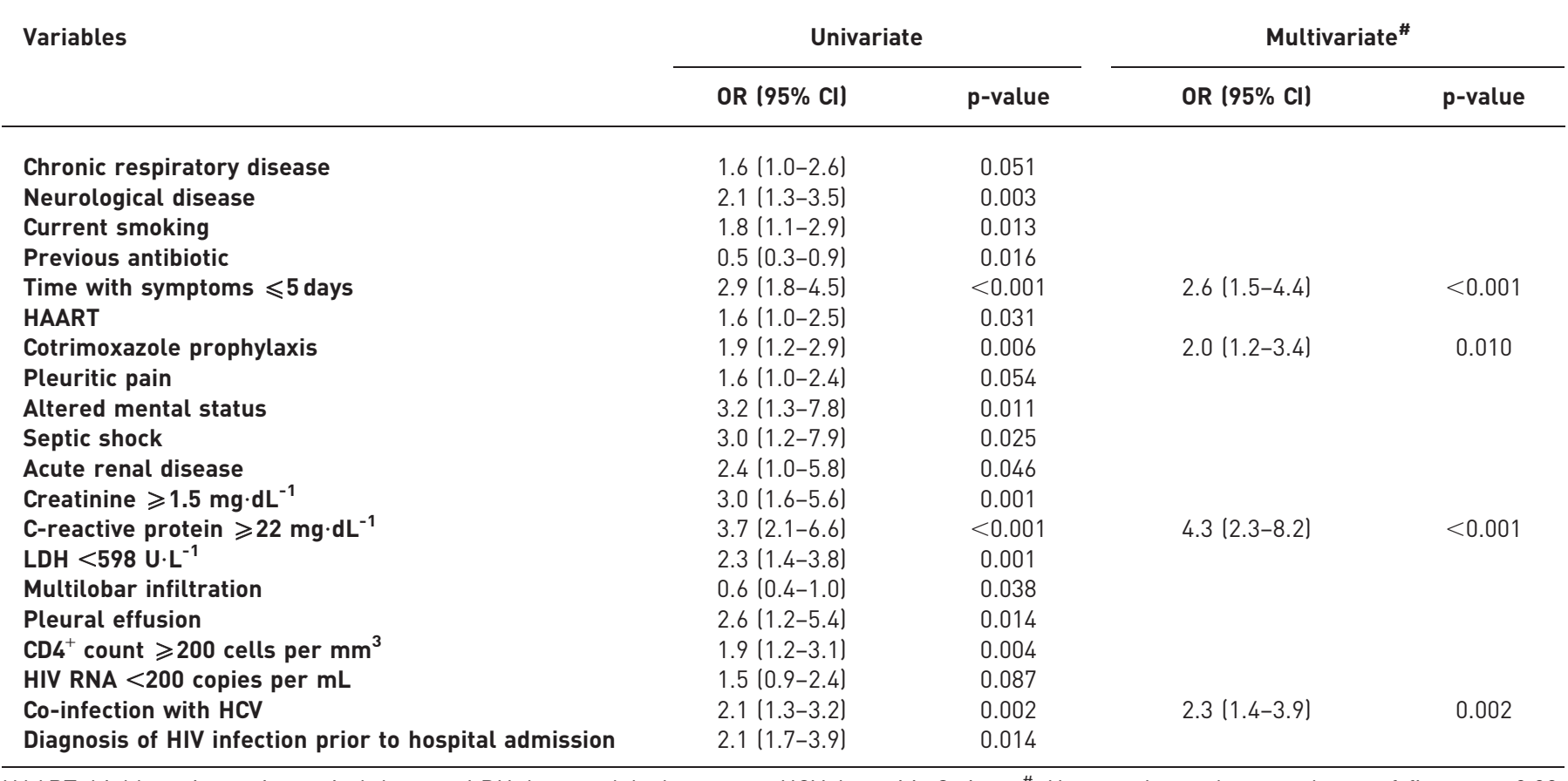

HAART: highly active antiretroviral therapy; LDH: lactate dehydrogenase; HCV: hepatitis C virus. * : Hosmer-Lemeshow goodness-of-fit test $p=0.89$.

TABLE 6 Significant univariate and multivariate logistic regression analyses of predictors for Pneumocystis jirovecii pneumonia in the HIV population

\begin{tabular}{|c|c|c|c|c|}
\hline Variables & \multicolumn{2}{|c|}{ Univariate } & \multicolumn{2}{|c|}{ Multivariate ${ }^{\#}$} \\
\hline Age per 5 years & $0.8(0.6-1.0)$ & 0.019 & & \\
\hline Chronic respiratory disease & $0.2(0.1-0.6)$ & 0.005 & & \\
\hline Neurological disease & $0.2(0.1-0.6)$ & 0.005 & & \\
\hline Time with symptoms $>5$ days & $4.8(2.1-10.7)$ & $<0.001$ & & \\
\hline HAART & $0.2(0.1-0.5)$ & $<0.001$ & & \\
\hline Cotrimoxazole prophylaxis & $0.2(0.1-0.4)$ & $<0.001$ & $0.1(0.04-0.5)$ & 0.003 \\
\hline Pleuritic pain & $0.4(0.2-0.9)$ & 0.023 & & \\
\hline Creatinine $<1.5 \mathrm{mg} \cdot \mathrm{dL}^{-1}$ & $2.8(0.8-9.4)$ & 0.095 & & \\
\hline C-reactive protein $<12 \mathrm{mg} \cdot \mathrm{dL}^{-1}$ & $3.2(1.5-6.9)$ & 0.002 & & \\
\hline HIV RNA $\geqslant 200$ copies per $\mathrm{mL}$ & $10.1(2.4-42.7)$ & 0.002 & & \\
\hline Co-infection with HCV & $0.3(0.2-0.7)$ & 0.005 & & \\
\hline $\begin{array}{l}\text { Diagnosis of HIV infection during the episode of } \\
\text { pneumonia }\end{array}$ & $6.2(3.1-12.4)$ & $<0.001$ & & \\
\hline
\end{tabular}


TABLE 7 Significant univariate and multivariate logistic regression analyses of predictors for 30-day mortality in the HIV population

\begin{tabular}{|c|c|c|c|c|}
\hline \multirow[t]{2}{*}{ Variable } & \multicolumn{2}{|c|}{ Univariate } & \multicolumn{2}{|c|}{ Multivariate ${ }^{\#}$} \\
\hline & OR $(95 \% \mathrm{Cl})$ & p-value & OR $(95 \% \mathrm{Cl})$ & p-value \\
\hline Cough & $0.4(0.2-1.0)$ & 0.054 & & \\
\hline Dyspnoea & $2.9(1.0-8.9)$ & 0.057 & & \\
\hline Altered mental status & $2.9(0.9-9.3)$ & 0.075 & & \\
\hline C-reactive protein $<12 \mathrm{mg} \cdot \mathrm{dL}^{-1}$ & $2.4(0.9-6.4)$ & 0.084 & & \\
\hline WBCs $\leqslant 4 \times 10^{12}$ per $L$ & $4.4(1.8-10.8)$ & 0.002 & & \\
\hline $\mathrm{LDH} \geqslant 598 \mathrm{U} \cdot \mathrm{L}^{-1}$ & $4.0(1.6-9.7)$ & 0.002 & $6.2(1.8-21.8)$ & 0.005 \\
\hline Multilobar infiltration & $3.5(1.4-8.8)$ & 0.008 & & \\
\hline Mechanical ventilation & $25.7(8.8-74.7)$ & $<0.001$ & $22.0(6.2-78.6)$ & $<0.001$ \\
\hline Appropriate antibiotic treatment & $0.1(0.04-0.2)$ & $<0.001$ & $0.1(0.03-0.4)$ & $<0.001$ \\
\hline
\end{tabular}

WBC: white blood cell; LDH: lactate dehydrogenase. " : Hosmer-Lemeshow goodness-of-fit test $p=0.48$.

Univariate and multivariate logistic regression analyses revealed several variables significantly associated with 30-day mortality (table 7).

The multivariate analysis showed that appropriate antibiotic treatment (OR 0.1, 95\% CI 0.03-0.4), LDH $\geqslant 598 \mathrm{U} \cdot \mathrm{L}^{-1}$ (OR 6.2, 95\% CI 1.8-21.8) and mechanical ventilation (OR 22.0, 95\% CI 6.2-78.6) were independently associated with 30-day mortality. The goodness-of-fit of the model tested with the HosmerLemeshow test revealed adequate model fit $(\mathrm{p}=0.48)$.

\section{Discussion}

The most important findings of our study are: 1) S. pneumoniae is the most prevalent isolate in HIVinfected patients with pneumonia, followed by P. jirovecii and mixed aetiology; 2) P.jirovecii is more frequent than S. pneumoniae in patients with $<200 \mathrm{CD}^{+}$cells per $\mathrm{mm}^{3}$ and the inverse is true in patients with detectable plasma HIV RNA load ( $\geqslant 200$ copies per $\mathrm{mL}$ ); 3 ) an acute clinical course ( $\leqslant 5$ days), the use of cotrimoxazole prophylaxis, a high level of C-reactive protein $\left(\geqslant 22 \mathrm{mg} \cdot \mathrm{dL}^{-1}\right)$ and $\mathrm{HCV}$ co-infection are risk factors for bacterial CAP; 4 ) lower WBC count $\left(\leqslant 4 \times 10^{12}\right.$ per L), high serum LDH level $\left(\geqslant 598 \mathrm{U} \cdot \mathrm{L}^{-1}\right)$, multilobar infiltration, female sex, current smoking and the use of cotrimoxazole prophylaxis are factors associated with a higher risk of PCP; and 5) appropriate antibiotic treatment, high level of LDH $\left(\geqslant 598 \mathrm{U} \cdot \mathrm{L}^{-1}\right)$ and, particularly, the need for mechanical ventilation are predictors of 30 -day mortality.

In this large series of HIV patients with CAP, we found that $50 \%$ were not receiving HAART therapy and $37 \%$ of patients had $\mathrm{CD}^{+}$counts $<200$ per $\mathrm{mm}^{3}$. This is because there was a significant proportion of late presenters $(62 \%)$ and patients with poor adherence to HAART and PCP prophylaxis.

In addition, a large percentage of patients had respiratory, neurological or hepatic comorbidities. Nevertheless, S. pneumoniae was the most frequent microorganism isolated. P. jirovecii was the second causal microorganism, thus indicating that we still must consider this microbial aetiology. The third aetiological cause was mixed pneumonia and this mainly included a combination of bacteria plus a respiratory virus. Importantly, we found only four cases of Pseudomonas aeruginosa and other multiresistant microorganisms were not isolated. CORDERO et al. [11] found that S. pneumoniae was the most frequent aetiology, followed by P. aeruginosa (20\%) and H. influenzae (14\%). Possibly, the observed discrepancy in the P. aeruginosa rate is possibly due to different HIV populations, as the study by CORDERO et al. [11] was published more than 12 years ago. In a recent extensive review article [2], $H$. influenzae is quoted as the second bacterial cause in this population while we found only a few cases of this infection.

More recent reports from the EuroSIDA cohort do no report microbial aetiology [12]. The originality of our report is the inclusion of bacterial and P. jirovecii causes of CAP together, as we believe this corresponds to the clinical reality when dealing with HIV-infected patients with pulmonary infiltrates admitted to hospital from the community.

By dividing patients according to $\mathrm{CD}^{+}$count, we find that $P$. jirovecii was the most frequent microorganism in patients with $<200 \mathrm{CD}^{+}$cells per $\mathrm{mm}^{3}$ (online supplementary table 1), followed by S. pneumoniae and 
mixed aetiology. These findings are not surprising and in line with other studies describing that HIVinfected patients with low $\mathrm{CD}^{+}$counts have a higher chance of suffering a PCP. However, in this population $\left(<200 \mathrm{CD}^{+}\right.$cells per $\left.\mathrm{mm}^{3}\right)$, we should still consider S. pneumoniae and mixed aetiologies, and cover these microorganisms accordingly.

We also stratified patients according to plasma HIV RNA load (online supplementary table 2). Although the level of HIV RNA copies could not discriminate S. pneumoniae, P. jirovecii was rare in patients on HAART $(<200$ copies per $\mathrm{mL})$ who were virologically suppressed, regardless their level of $\mathrm{CD} 4^{+}$cell count. This the first study performing this kind of analysis. This observation might be useful for clinicians to guide initial antibiotic treatments in the emergency department.

Despite all these findings, in clinical practice, it is difficult to discriminate between bacterial CAP and PCP, and clinicians often cover both types of microorganisms until microbiological results are available. Among the reasons for this there are surely the lack of information on the immunological status of HIV patients and the clinicians' apprehension of undiagnosed HIV cases. Considering these aspects, it would be useful to have clinical and biological predictors to distinguish between bacterial CAP and PCP, and to support the initial decision on empirical antibiotic therapy while HIV infection is still not clear.

In our study, multivariate analysis showed some predictors of bacterial CAP: acute clinical course ( $\leqslant 5$ days), the use of cotrimoxazole prophylaxis, a high level of C-reactive protein $\left(\geqslant 22 \mathrm{mg} \cdot \mathrm{dL}^{-1}\right)$ and HCV co-infection. Furthermore, we found that female sex, not smoking, the lack of cotrimoxazole prophylaxis, leukopenia, $\mathrm{LDH} \geqslant 598 \mathrm{U} \cdot \mathrm{L}^{-1}$ and multilobar infiltration were factors associated with a higher risk of PCP. Among these, the most important factors were serum LDH and multilobar involvement. Previous articles have emphasised the predictive value of serum LDH in PCP. A recent Swiss report [13] found a sensitivity of $100 \%$ and a specificity of $58 \%$ using $\mathrm{LDH}>250 \mathrm{U} \cdot \mathrm{L}^{-1}$. In our study, the combination of both multilobar involvement and $\mathrm{LDH} \geqslant 598 \mathrm{U} \cdot \mathrm{L}^{-1}$ gave very good operational values (76\% sensitivity and $89 \%$ specificity) and could support the medical decision to empirically cover PCP in the case of a concomitant clinical suspicion of HIV infection. This clinical dilemma may be resolved in the near future with the use of rapid molecular point-of-care microbiological testing.

Interestingly, we found that current or past smoking was independently associated with a lower risk of P. jirovecii. A recent report by GoRDIN et al. [14] found, in a follow-up study, that actively smoking HIVinfected patients who had interrupted HAART had an increased risk of bacterial pneumonia. The effect of smoking on the development of PCP is consistent with data from by SHIVjI et al. [15] who showed that Sadenosylmethionine, a critical cellular metabolic intermediate necessary for P.jirovecii growth, is significantly reduced by nicotine exposure. This finding clearly explains the reduced rate of PCP among smoking AIDS patients [15].

In this study, the observed 30-day mortality was only 7\%, while only 63 (19\%) patients required ICU admission and $49(15 \%)$ mechanical ventilation. Our recent fatality rate is possibly better than that we expected and clearly driven by PCP, as also shown in a previous multicentre Spanish HIV series (2000-2004) describing an overall mortality rate of $15 \%$ for PCP (rising to $80 \%$ in patients requiring mechanical ventilation), with very similar ICU (21\%) and mechanical ventilation rates (16\%) [16]. The mortality in our series dramatically increases with PCP, being more than double that of bacterial CAP (14\% versus $6 \%$ ) (table 3 ). The only three variables independently associated with mortality in the overall HIV population were: appropriate antibiotic treatment (OR 0.1, 95\% CI 0.03-0.4); LDH $\geqslant 598 \mathrm{U} \cdot \mathrm{L}^{-1}$ (OR 6.2, 95\% CI 1.8-21.8), which was clearly correlated to PCP (table 5); and mechanical ventilation (OR 22.0, 95\% CI 6.2-78.6).

Some limitations of our study must be pointed out. First, it included only patients visiting our hospital. Second, the complete diagnostic work-up and microbiological sampling could not be fully applied in all patients. This means that some viral or atypical causes of CAP could have been missed. However, all patients were enrolled in the study prospectively and consecutively, thus lending consistency to our data.

In conclusion, S. pneumoniae and P. jirovecii are the most frequent microorganisms in HIV patients with CAP. There are several predictors that may help clinicians to distinguish between bacterial and PCP.

\section{Acknowledgements}

This study was presented as a part of a thesis in the XI Master of AIDS of the University of Barcelona, Barcelona, Spain. Thanks to M. Tuset Creus (University of Barcelona, Barcelona, Spain) for their support.

\section{References}

1 Feikin DR, Feldman C, Schuchat A, et al. Global strategies to prevent bacterial pneumonia in adults with HIV disease. Lancet Infect Dis 2004; 4: 445-455.

2 Benito N, Moreno A, Miro JM, et al. Pulmonary infections in HIV-infected patients: an update in the 21st century. Eur Respir J 2012; 39: 730-745. 
Madeddu G, Porqueddu EM, Cambosu F, et al. Bacterial community acquired pneumonia in HIV-infected inpatients in the highly active antiretroviral therapy era. Infection 2008; 36: 231-236.

4 Hirschtick R, Glassroth J, Jordan M, et al. Bacterial pneumonia in persons infected with the human immunodeficiency virus. N Engl J Med 1995; 333: 845-851.

5 Phair J, Munoz A, Detels R, et al. The risk of Pneumocystis carinii pneumonia among men infected with human immunodeficiency virus type 1. Multicenter Aids Cohort Study Group. N Engl J Med 1990; 322: $161-165$.

6 Llibre JM, Revollo B, Vanegas S, et al. Pneumocystis jirovecii pneumonia in HIV-1-infected patients in the lateHAART era in developed countries. Scand J Infect Dis 2013; 45: 635-644.

7 Napoli AM, Maughan B, Murray R, et al. Use of the relationship between absolute lymphocyte count and cd4 count to improve earlier consideration of Pneumocystis pneumonia in HIV-positive emergency department patients with pneumonia. J Emerg Med 2013; 44: 28-35.

8 Antinori A, Coenen T, Costagiola D, et al. Late presentation of HIV infection: a consensus definition. HIV Med 2011; 12: 61-64.

9 Mandell LA, Wunderink RG, Anzueto A, et al. Infectious Diseases Society Of America/American Thoracic Society consensus guidelines on the management of community-acquired pneumonia in adults. Clin Infect Dis 2007; 44: Suppl. 2, S27-S72.

10 Hosmer D, Lemeshow S. Applied Logistic Regression, New York, Wiley, 1989.

11 Cordero E, Pachon J, Rivero A, et al. Community-acquired bacterial pneumonia in human immunodeficiency virus-infected patients: validation of severity criteria. The Grupo Andaluz Para El Estudio De Las Enfermedades Infecciosas. Am J Respir Crit Care Med 2000; 162: 2063-2068.

12 Sogaard OS, Reekie J, Ristola M, et al. Severe bacterial non-AIDS infections in HIV-positive persons: incidence rates and risk factors. $J$ Infect 2013; 66: 439-446.

13 Vogel M, Weissgerber P, Goeppert B, et al. Accuracy of serum LDH elevation for the diagnosis of Pneumocystis jiroveci pneumonia. Swiss Med Wkly 2011; 141: W13184.

14 Gordin FM, Roediger MP, Girard PM, et al. Pneumonia in HIV-infected persons: increased risk with cigarette smoking and treatment interruption. Am J Respir Crit Care Med 2008; 178: 630-636.

15 Shivji M, Burger S, Moncada CA, et al. Effect of nicotine on lung S-adenosylmethionine and development of Pneumocystis pneumonia. J Biol Chem 2005; 280: 15219-15228.

16 Varez-Martinez MJ, Moreno A, Miro JM, et al. Pneumocystis jirovecii pneumonia in Spanish HIV-infected patients in the combined antiretroviral therapy era: prevalence of dihydropteroate synthase mutations and prognostic factors of mortality. Diagn Microbiol Infect Dis 2008; 62: 34-43. 